\title{
Atmosphere parameters of four K-giants obtained by spectrum fitting
}

\author{
J. Krtička and V. Štefl \\ Dept. of Theor. Phys. and Astrophysics, Faculty of Science, Masaryk University, Kotlářská 2, CZ-611 37 Brno, Czech Republic
}

Received April 30, 1998; accepted March 2, 1999

\begin{abstract}
Basic parameters of the atmospheres (i.e., $T_{\text {eff }}$, $\left.\log g,[A / H], v_{\text {turb }}\right)$ of four late type giant stars $\alpha$ Ari, $\alpha$ Boo, $\alpha$ Cas, $\beta$ Gem are obtained by matching synthetic spectra to observed ones. We used model atmosphere to compute the synthetic spectrum. The parameters of the model atmosphere and of the synthetic spectrum are fitted to match the observed spectrum, accelerated by means of a proposed numerical tool.
\end{abstract}

Key words: stars: fundamental parameters - stars: abundances - stars: $\alpha$ Ari; $\alpha$ Boo; $\alpha$ Cas; $\beta$ Gem

\section{Introduction}

Knowledge of the astrophysical parameters of the stellar atmospheres (i.e., $T_{\text {eff }}, \log g,[A / H], v_{\text {turb }}$ ) of any given star is crucial for many fields of modern astrophysics. Recent developments in model atmosphere computation and significant increase in computer speeds make a global approach to this problem possible. Parameters of the model atmosphere and the synthetic spectra can be fit to match observed spectra.

Recently published works on spectrum fitting (e.g. Cuisinier et al. 1994) apply a pretabulated grid of synthetic spectra (see Barbuy et al. 1990; Cayrel et al. 1991 for spectra with lower resolving power, Chavez et al. 1997 for spectra with higher resolving power). Our method does not employ any pretabulated grid of synthetic spectra, but we do compute synthetic spectra using the appropriate model atmosphere in different wavelength intervals. A spectral region less crowded by lines (in the red part of spectrum) leads to more accurate determination of the continuum position. A similar approach was proposed by some authors with automatic codes: MERSEN (Cowley 1996), SME (Valenti \& Piskunov 1996) and some others.

\section{Observations}

The list of spectra applied in this article is given in Table 1. We used the Reticon spectra described by Horn et al. (1996) for each star in our list. The spectrograms were acquired at the coudé focus of the 2.0-m telescope of the Astronomical Institute of the Czech Academy of Sciences, Ondřejov. The reduction of these spectra was carried out by the program SPEFO, developed by Horn (Škoda 1996). For $\alpha$ Cas we employed the spectra fully described by Stefl $(1985,1994)$. The spectra were obtained with the 2.0-m telescope of the Astronomical Institute, Ondřejov. We also employed spectra from the Arcturus atlas (Wallace \& Hinkle 1996). For $\alpha$ Ari, we utilized spectra from the library of high and mid-resolution spectra (Montes et al. 1997). Finally, for $\beta$ Gem and $\alpha$ Cas we acquired spectra at the observatory of Masaryk University at Kravíhora, Brno by the CCD Jobin Yvon spectrometer (with $1200 \mathrm{gr} / \mathrm{mm}$ blazed grating) attached to the $0.6 \mathrm{~m}$ telescope.

Table 1. Observation applied in this article

\begin{tabular}{ccccc}
\hline Star & $\begin{array}{c}\text { Wavelength } \\
\text { region }[\AA]\end{array}$ & $\begin{array}{c}\text { Resolving } \\
\text { power }\end{array}$ & Source & Notes \\
\hline$\alpha$ Ari & $6300-6700$ & 13000 & Horn et al. (1996) & \\
& $5765-6010$ & & Montes et al. (1997) & \\
$\alpha$ Boo & $6300-6700$ & 13000 & Horn et al. (1996) & \\
& $8690-9260$ & & Wallace \& Hinkle (1996) & \\
$\alpha$ Cas & $6300-6700$ & 13000 & Horn et al. (1996) & \\
& $5740-6350$ & 50000 & Sttefl (1985, 1994) & 1 \\
& $6300-6650$ & 50000 & Štefl (1985, 1994) & 1 \\
& $6300-6700$ & 7000 & Kravíhora observatory & 1 \\
$\beta$ Gem & $6300-6700$ & 13000 & Horn et al. (1996) & \\
& $5000-5350$ & 6000 & Kravíhora observatory & \\
\hline Notes: 1 Used only in the Sect. 5. & &
\end{tabular}




\section{Numerical methods}

\subsection{Model atmosphere}

A model atmosphere was obtained firstly with the ATLAS9 program (Kurucz 1993) adapted for Sun OS by M. Lemke. According to numerical simulation of stellar convection (Abbett et al. 1997) we have selected $l / H=1.5$, and for models we put $v_{\text {turb }}=2.0 \mathrm{~km} \mathrm{~s}^{-1}$.

Second, we applied the POSMARCS grid of models (Plez 1997) described in some details by Bessell et al. (1998) and based on SOSMARCS models (Plez et al. 1992). The microturbulent velocity was chosen as $v_{\text {turb }}=$ $2.0 \mathrm{~km} \mathrm{~s}^{-1}$.

\subsection{Synthetic spectra}

The synthetic spectra are calculated by the program SYNSPEC36 (Hubeny et al. 1994). Several modifications of this program have been made to adapt the program to spectrum synthesis of cool stars. The most important modifications are the inclusion of atoms with $30<Z \leq 90$ and the inclusion of selected transitions of some diatomic molecules.

The polynomial partition function approximations for atoms with $30<Z \leq 90$ are adopted from Irwin (1981). The molecular constants are taken from Huber \& Herzberg (1979), while the line list is taken from Kurucz's CD-ROM (1993). Atomic line data for the spectrum synthesis are adopted from VALD (Piskunov et al. 1995). The Hubeny's program ROTIN3 computes rotational and instrumental convolution.

\subsection{Optimization}

Two steps (computation of model atmosphere and synthetic spectrum) are repeated to match the observed spectrum. A non-linear method optimizes the model parameters and/or synthetic spectrum parameters. To avoid numerical problems during the calculation of gradient we have chosen a simplex method.

We used two different methods for the calculation of the optimization criterion (see below).

\subsubsection{Direct comparison}

The computed synthetic flux is convolved with the rotational and instrumental kernel function. The final synthetic spectrum is obtained in a denser wavelength grid than the observed one (because of more accurate computation of the convolution), so a spline interpolation is applied to obtain a uniform wavelength grid. The optimization criterion is

$\sigma^{2}=\sum_{n}\left(H_{n}^{\mathrm{obs}}-H_{n}^{\mathrm{syn}}\right)^{2}$ where $H$ is the line profile and subscripts obs and syn denote observed and synthetic spectrum, respectively.

\subsubsection{Comparison applying Fourier transform}

Since the rotational velocity $v \sin i$ and the so called macroturbulent velocity are not important quantities for stellar atmosphere, we applied the Fourier transform method for adopting the basic parameters of stellar atmosphere. Lowering the number of independent variables significantly decreases the computer time spent for each fitting, and does not require knowledge of the detailed shape of the instrumental profile.

An ideal observed spectrum $H_{\text {ideal }}$ for slowly rotating stars is the convolution of the flux emergent from the star $H_{\mathrm{em}}$ and the rotational profile $I_{\text {rot }}$ (merged with the profile of the macroturbulent velocity); let us write according to Gray (1992)

$H_{\text {ideal }}=H_{\mathrm{em}} * I_{\mathrm{rot}}$.

Likewise, the final observed spectrum $H_{\text {inst }}$ is a convolution of an ideal observed spectrum and instrumental profile $I_{\text {inst }}$

$H_{\mathrm{obs}}=H_{\text {ideal }} * I_{\text {inst }}$.

Applying known features of the convolution we can now write

$H_{\mathrm{obs}}=H_{\mathrm{em}} * I$,

where $I$ is the convolution of rotational and instrumental profile

$I=I_{\text {rot }} * I_{\text {inst }}$.

Fourier transform of Eq. (4) gives

$\mathcal{H}_{\mathrm{obs}}=\mathcal{H}_{\mathrm{em}} \mathcal{I}$

where $\mathcal{H}_{\mathrm{obs}}, \mathcal{H}_{\mathrm{em}}$ and $\mathcal{I}$ denote the Fourier transform of the corresponding quantities. From this equation we can now determine the instrumental profile $I$ as

$I \equiv \mathcal{F}(\mathcal{I})=\mathcal{F}\left(\mathcal{H}_{\mathrm{obs}} / \mathcal{H}_{\mathrm{em}}\right)$

where $\mathcal{F}$ denotes the Fourier transform. A typical result of such a calculation is a function which has non-zero values only in a very small interval. This result is demonstrated in Fig. 1 and can be used for the optimization, as described below.

The computed synthetic spectrum is spline interpolated to $n_{\text {obs }}$ wavelength points of the observed spectrum. Then, FFT is performed on both observed and synthetic spectrum to obtain $\mathcal{H}_{\mathrm{obs}}$ and $\mathcal{H}_{\mathrm{em}}$, where $\mathcal{H}_{\mathrm{em}}$ now denotes the Fourier transform of the synthetic spectrum. Equation (6) is consequently used to compute $\mathcal{I}$, the Fourier transform of convolution of instrumental and rotational profiles. Furthermore, backward FFT is performed 




Fig. 1. A sample of the Fourier transformation of the convolution of tentative instrumental and rotational profiles. The solid line is a part of that Fourier transformation (for $\beta \mathrm{Gem}$ ) computed with optimum parameters (given in Table 2) and the dashed line is a part of that Fourier transform with temperature shifted about $500 \mathrm{~K}$. The peak (around $n=0$ ) represents the Fourier transform of the convolution of the rotational and instrumental profile. For optimum parameters this peak is higher. Furthermore, beginning from a certain point (in this case $n \gtrsim 10$ ) values of $\mathcal{I}$ computed with shifted temperature are higher than values computed with correct temperature. If we could compute an accurate synthetic spectrum, then the values outside the peak would be equal to zero

on derived profile $\mathcal{I}$, applying Eq. (7). The final optimization criterion is computed in the form of

$\sigma^{2}=\sum_{n=n_{\text {inst }}}^{n_{\mathrm{obs}}-n_{\text {inst }}} I_{n}^{2}$,

where $2 \cdot n_{\text {inst }}$ is the number of points where the profile is significantly non-zero. Globally, the tentative instrumental profile $I$ is computed from Eq. (7), where $\mathcal{H}_{\mathrm{em}}$ is substituted by the Fourier transform of the synthetic spectrum and $\mathcal{H}_{\mathrm{obs}}$ is the Fourier transform of the observed spectrum. Different values of $\sigma^{2}$ can be obtained by varying the parameters of the synthetic spectrum. The parameters of spectra with the lowest $\sigma^{2}$ are close to genuine atmosphere parameters of the star.

To indicate the quality of the fit, we used the quantity $\bar{\sigma}$, defined as

$\bar{\sigma}=\sqrt{\frac{\sigma^{2}}{n_{\mathrm{obs}}}}$

where $n_{\text {obs }}$ is number of points of the observed spectrum.

\section{Results}

For spectrum synthesis the only molecule added was CN $\left(\mathrm{A}^{2} \Pi-\mathrm{X}^{2} \Sigma\right)$, which was applied in the infrared part of Arcturus spectrum. The mean parameters (the effective temperature, the gravity, the microturbulent velocity and the metallicity) obtained in this work are given in Table 2.
Table 2. Derived atmosphere parameters of program stars

\begin{tabular}{ccccc}
\hline star & $T_{\text {eff }}[\mathrm{K}]$ & $\log g$ & $v_{\text {turb }}\left[\mathrm{km} \mathrm{s}^{-1}\right]$ & {$[A / H]$} \\
\hline$\alpha$ Ari & $4590 \pm 100$ & $2.4 \pm 0.1$ & $2.4 \pm 0.2$ & $-0.15 \pm 0.16$ \\
$\alpha$ Boo & $4390 \pm 90$ & $2.0 \pm 0.2$ & $1.6 \pm 0.2$ & $-0.27 \pm 0.05$ \\
$\alpha$ Cas & $4520 \pm 50$ & $1.83 \pm 0.03$ & $2.25 \pm 0.15$ & $-0.04 \pm 0.01$ \\
$\beta$ Gem & $4990 \pm 30$ & $2.92 \pm 0.06$ & $2.0 \pm 0.1$ & $-0.03 \pm 0.03$ \\
\hline
\end{tabular}

\section{1. $\alpha$ Ari}

Results of the FFT optimization described in Sect. 3.3.2 in each wavelength interval are given in Table 3 . Parameters of $\alpha$ Ari determined by other authors are given in Table 4 .

Table 3. Derived atmosphere parameters of $\alpha$ Ari

\begin{tabular}{ccccccc}
\hline $\begin{array}{c}\text { interval } \\
{[\AA]}\end{array}$ & $\begin{array}{c}T_{\text {eff }} \\
{[\mathrm{K}]}\end{array}$ & $\log g$ & $\begin{array}{c}v_{\text {turb }} \\
{\left[\mathrm{km} \mathrm{s}^{-1}\right]}\end{array}$ & {$[A / H]$} & model & $\bar{\sigma}$ \\
\hline $6300-6700$ & 4700. & 2.52 & 2.57 & -0.003 & ATLAS9 & 0.056 \\
$6300-6700$ & 4670. & 2.49 & 2.57 & -0.024 & POSMARCS & 0.057 \\
$5765-6010$ & 4500. & 2.33 & 2.30 & -0.278 & POSMARCS & 0.027 \\
\hline
\end{tabular}

Table 4. Available determinations of atmosphere parameters of $\alpha$ Ari

\begin{tabular}{cccc}
\hline$T_{\text {eff }}[\mathrm{K}]$ & $\log g$ & {$[\mathrm{Fe} / \mathrm{H}]$} & literature \\
\hline 4710 & 2.51 & -0.25 & Lambert \& Ries (1981) \\
4582 & 1.38 & -0.29 & Gratton et al. (1982) \\
4480 & 2.57 & -0.25 & McWilliam (1990) \\
4425 & 1.65 & -0.21 & Luck \& Challener (1995) \\
\hline
\end{tabular}

A comparison of the results indicated by the program ATLAS9 and POSMARCS shows negligible differences.

\section{2. $\alpha$ Boo}

The derived parameters obtained in each wavelength interval are summarized in Table 5. The spectra from Wallace \& Hinkle (1996) were fitted using the direct method (Sect. 3.3.1), and spectra from Horn et al. (1996) were fitted by means of the FFT method (Sect. 3.3.2). The parameters finally obtained are given in Table 2, except the rotational velocity which is equal to $6.4 \pm 0.7 \mathrm{~km} \mathrm{~s}^{-1}$.

The parameters of $\alpha$ Boo taken from the quoted articles are summarized in Table 6 . The metallicity obtained in this work is slightly higher than that determined by other authors.

\section{3. $\alpha$ Cas}

Results of the FFT optimization in each interval are given in Table 7. Parameters of $\alpha$ Cas determined by other authors are given in Table 8. 


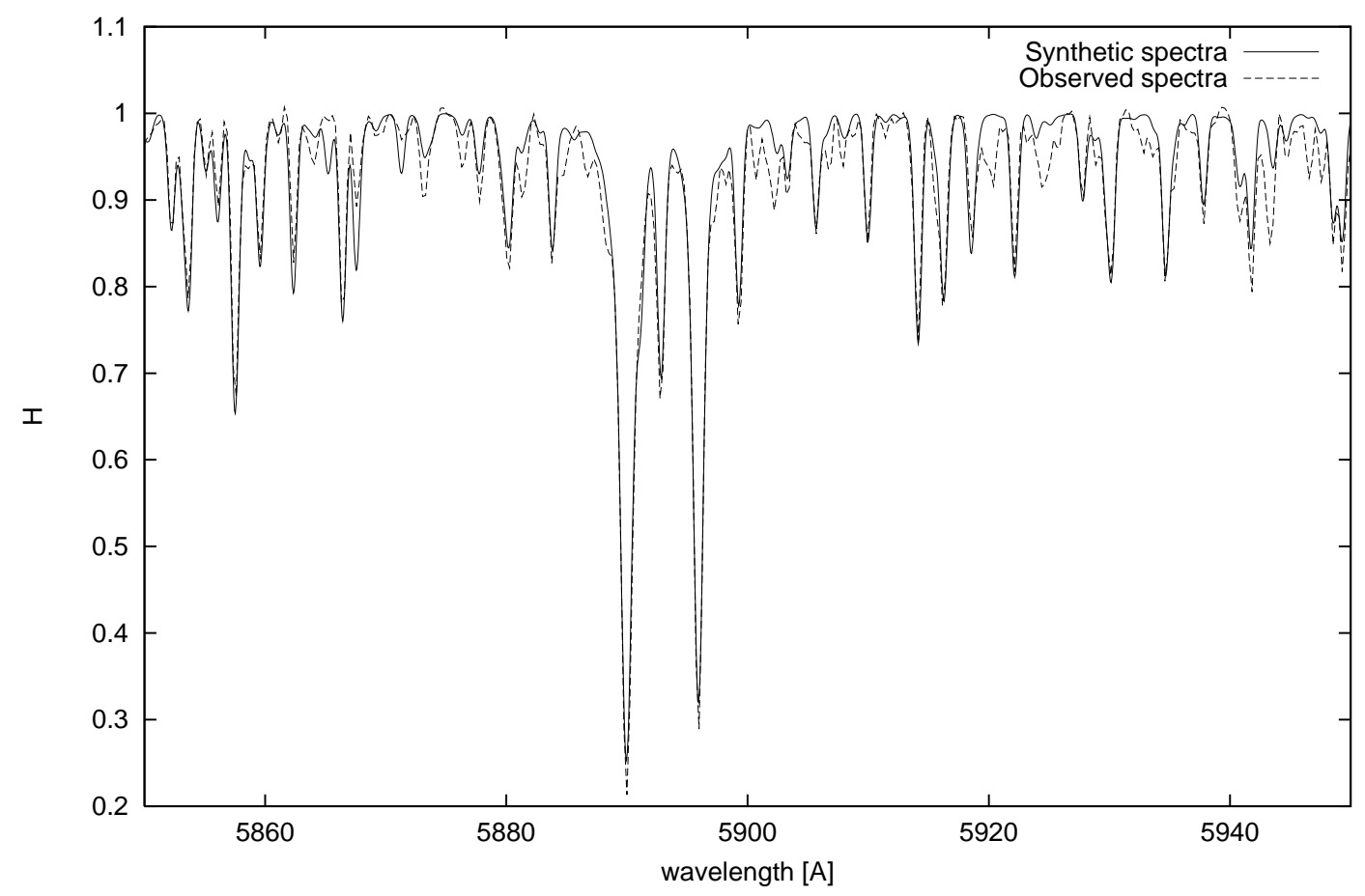

Fig. 2. Result of the optimization of $\alpha$ Ari using the model ATLAS9 and the observed spectrum adopted from the catalogue of Montes et al. (1997). Solid line - observed spectra, dashed line - synthetic spectra

Table 5. Derived atmosphere parameters of $\alpha$ Boo

\begin{tabular}{cccccccc}
\hline $\begin{array}{c}\text { interval } \\
{[\AA]}\end{array}$ & $\begin{array}{c}T_{\text {eff }} \\
{[\mathrm{K}]}\end{array}$ & $\log g$ & $\begin{array}{c}v_{\text {turb }} \\
{\left[\mathrm{km} \mathrm{s}^{-1}\right]}\end{array}$ & {$[A / H]$} & $v \sin i$ & model & $\bar{\sigma}$ \\
\hline $\left.\mathrm{km} \mathrm{s}^{-1}\right]$ & & \\
\hline $9170-9260$ & 4600 & 2.30 & 1.48 & -0.291 & 0.5 & POSMARCS & 0.074 \\
$9090-9180$ & 4470 & 2.35 & 1.51 & -0.155 & 6.6 & POSMARCS & 0.063 \\
$9000-9100$ & 4423 & 1.71 & 1.66 & -0.234 & 8.1 & POSMARCS & 0.055 \\
$8920-9010$ & 4330 & 1.53 & 1.25 & -0.367 & 6.5 & POSMARCS & 0.016 \\
$8850-8930$ & 4410 & 1.53 & 1.70 & -0.461 & 5.9 & POSMARCS & 0.016 \\
$8770-8850$ & 4400 & 2.50 & 2.03 & -0.256 & 5.3 & POSMARCS & 0.022 \\
$8690-8780$ & 4350 & 1.64 & 1.66 & -0.232 & 5.9 & POSMARCS & 0.032 \\
$7300-7700$ & 4200 & 1.96 & 2.89 & -0.246 & - & ATLAS9 & 0.049 \\
\hline
\end{tabular}

Table 6. Parameters of $\alpha$ Boo, available in the quoted articles

\begin{tabular}{cccc}
\hline$T_{\text {eff }}[\mathrm{K}]$ & $\log g[\mathrm{Fe} / \mathrm{H}]$ & literature \\
\hline 4500 & 2.01 & -0.56 & Lambert \& Ries (1981) \\
4421 & 0.98 & -0.48 & Gratton et al. (1982) \\
4345 & 1.50 & -0.38 & Gratton \& Ortolani (1986) \\
4235 & 1.7 & -0.60 & Leep et al. (1987) \\
4300 & 2.00 & -0.69 & Fernandez-Villacanas et al. (1990) \\
4280 & 2.19 & -0.60 & McWilliam (1990) \\
4330 & 2.10 & -0.58 & Brown \& Wallerstein (1992) \\
4280 & 1.30 & -0.54 & McWilliam \& Rich (1994) \\
4300 & 1.50 & -0.47 & Sneden et al. (1994) \\
\hline
\end{tabular}

\section{4. $\beta$ Gem}

Results of optimization in individual intervals are given in Table 9 . The parameters of $\beta$ Gem available in the quoted literature are given in Table 10 .
Table 7. Derived atmosphere parameters of $\alpha$ Cas. Spectra in the first row are taken from Horn et al. (1996), and the rest is taken from Štefl $(1985,1994)$

\begin{tabular}{ccccccc} 
interval $[\AA]$ & $T_{\text {eff }}[\mathrm{K}]$ & $\log g$ & $v_{\text {turb }}\left[\mathrm{km} \mathrm{s}^{-1}\right]$ & {$[A / H]$} & model & $\bar{\sigma}$ \\
\hline $6300-6700$ & 4500 & 1.81 & 2.36 & -0.026 & ATLAS9 & 0.045 \\
$6040-6200$ & 4650 & 1.85 & 1.92 & -0.035 & POSMARCS & 0.048 \\
$6200-6400$ & 4480 & 1.82 & 2.34 & -0.038 & POSMARCS & 0.063 \\
$5800-6000$ & 4530 & 1.88 & 2.29 & -0.039 & POSMARCS & 0.064 \\
$5740-5800$ & 4490 & 1.85 & 2.16 & -0.063 & POSMARCS & 0.064 \\
\hline
\end{tabular}

Table 8. Parameters of $\alpha$ Cas obtained from the literature

\begin{tabular}{cccc}
\hline$T_{\text {eff }}[\mathrm{K}]$ & $\log g$ & {$[\mathrm{Fe} / \mathrm{H}]$} & literature \\
\hline 4755 & 2.01 & -0.18 & Lambert \& Ries (1981) \\
4710 & 1.12 & -0.03 & Gratton et al. (1982) \\
4610 & 2.71 & -0.09 & McWilliam (1990) \\
4520 & 1.72 & -0.22 & Štefl (1994) \\
\hline
\end{tabular}

Table 9. Derived atmosphere parameters of $\beta$ Gem

\begin{tabular}{ccccccc}
\hline interval $[\AA]$ & $T_{\text {eff }}[\mathrm{K}]$ & $\log g$ & $v_{\text {turb }}\left[\mathrm{km} \mathrm{s}^{-1}\right]$ & {$[A / H]$} & model & $\bar{\sigma}$ \\
\hline $6300-6700$ & 5000 & 2.88 & 1.98 & -0.04 & ATLAS9 & 0.048 \\
$5000-5350$ & 4970 & 2.95 & 1.96 & -0.01 & ATLAS9 & 0.041 \\
\hline
\end{tabular}

Table 10. Parameters of $\beta$ Gem, taken from the literature

\begin{tabular}{clrc}
\hline$T_{\text {eff }}[\mathrm{K}]$ & $\log g$ & {$[\mathrm{Fe} / \mathrm{H}]$} & literature \\
\hline 5040 & 3.12 & 0.02 & Lambert \& Ries (1981) \\
4941 & 2.48 & 0.16 & Gratton et al. (1982) \\
4800 & 2.6 & -0.11 & Tomkin \& Lambert (1983) \\
4850 & 2.96 & -0.07 & McWilliam (1990) \\
4865 & 2.75 & -0.04 & Drake \& Smith (1991) \\
\hline
\end{tabular}





Fig. 3. A comparison of observed (solid line) and the best fitted synthetic spectra (dashed line) for $\alpha$ Boo in the infrared region. The observed spectrum is taken from the Arcturus atlas (Wallace \& Hinkle 1996) 


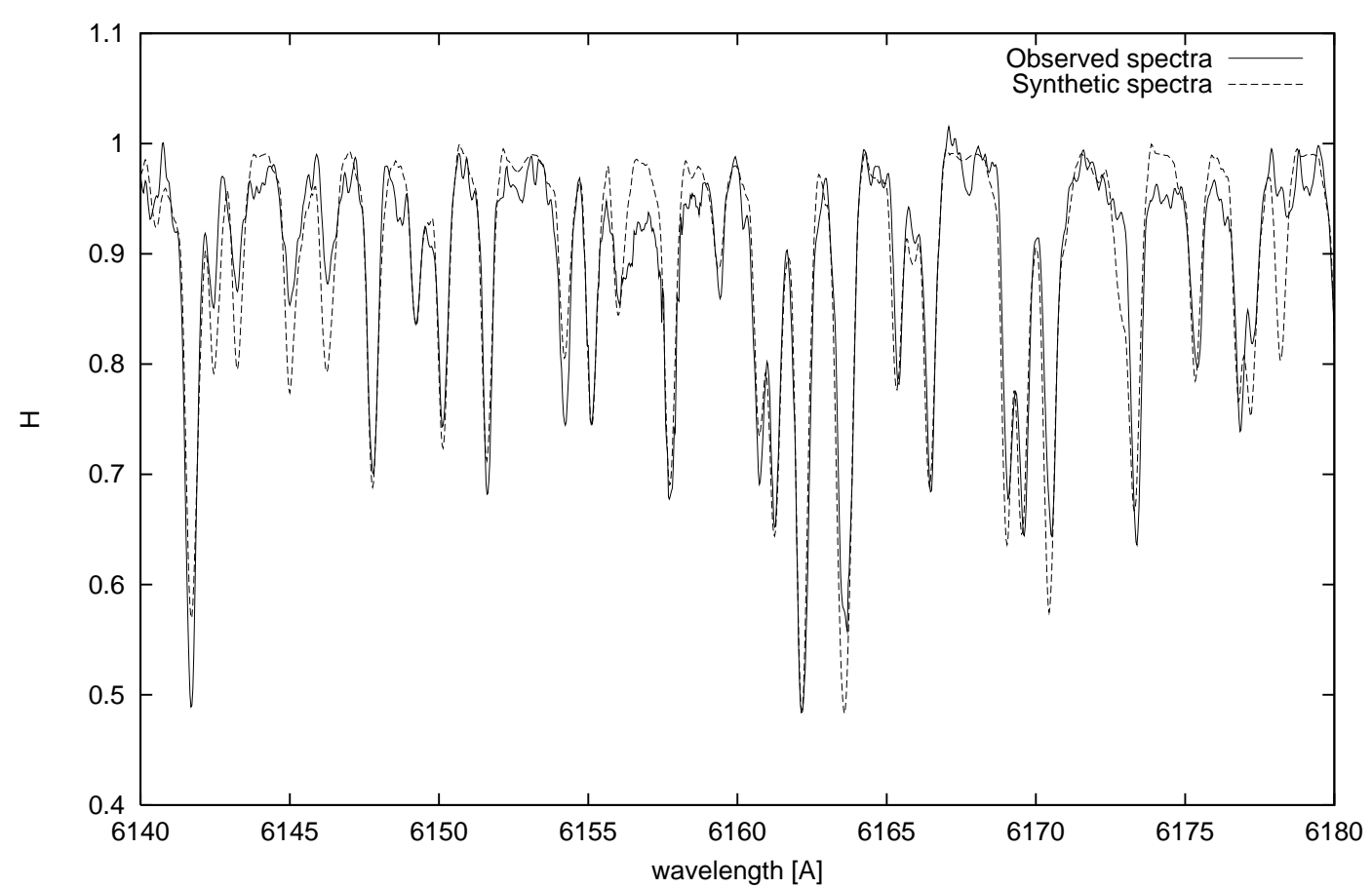

Fig. 4. A comparison of observed (solid line) and the best fitted synthetic spectra (dashed line) for $\alpha$ Cas. The upper observed spectrum is taken from Štefl $(1985,1994)$, the bottom one was adopted from Horn et al. (1996)

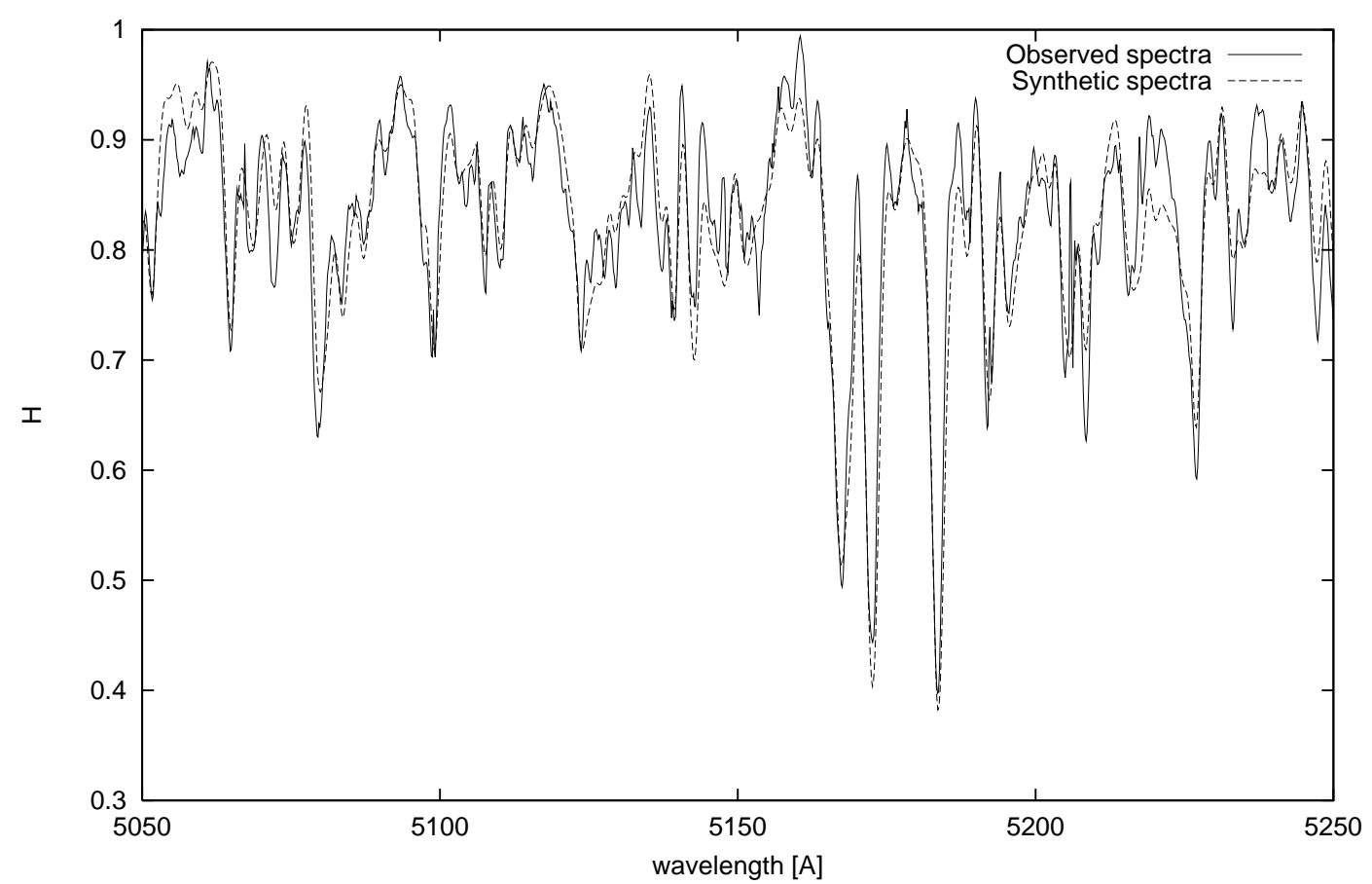

Fig. 5. A comparison of the observed (solid line) and the best fitted synthetic spectra (dashed line) for $\beta$ Gem. The observed spectrum was acquired at Kraví hora observatory 


\section{Spectra with different resolving powers}

Another interesting comparison, suggested by our referee, is the processing of spectra with different resolving powers. For such an investigation we selected $\alpha$ Cas. Information taken from a spectrum generally depends on resolving power, but an equivalent width does not, while our technique is in principle independent on the resolving power. Moreover, the correctness of the continuum location can be tested using spectra with different resolving powers. In Table 11 we summarized the results obtained in very similar wavelength intervals (approximately $6300 \AA-6700 \AA$ ) using spectra acquired with different resolving powers. From the table it turns out that the results obtained with different spectrograms (specially metallicity) do not depend on the resolving power.

Table 11. Parameters of $\alpha$ Cas obtained from spectra with different resolving powers. For this calculation we applied POSMARCS models

\begin{tabular}{cccccc}
\hline$R$ & $T_{\text {eff }}[\mathrm{K}]$ & $\log g$ & $v_{\text {turb }}\left[\mathrm{km} \mathrm{s}^{-1}\right]$ & {$[A / H]$} & $\bar{\sigma}$ \\
\hline 7000 & 4570 & 1.73 & 3.00 & -0.09 & 0.050 \\
13000 & 4510 & 1.78 & 2.37 & -0.10 & 0.045 \\
50000 & 4500 & 1.81 & 2.35 & -0.06 & 0.069 \\
\hline
\end{tabular}

\section{Conclusions}

The parameters of each star obtained in different wavelength intervals are close to each other, except for Arcturus, where we can find large differences in both effective temperature and gravity. One of the possible reasons may be that the infrared regions used in this work are not overly occupied by lines.

The effective temperatures obtained are in good agreement with that indicated by Gratton et al. (1982). Conversely, gravities are close to that derived by McWilliam (1990) and Lambert \& Ries (1981).

The value obtained for $[A / H]$ cannot be directly compared with that of $[\mathrm{Fe} / \mathrm{H}]$ obtained by other authors because our value of $[A / H]$ represents an averaged quantity, since the majority of lines are Fe-lines in the spectra of K-giants, the value of $[A / H]$ has to be close to $[\mathrm{Fe} / \mathrm{H}]$. Moreover, small discrepancy between $[\mathrm{Fe} / \mathrm{H}]$ available in the quoted literature and the results presented in this paper may be caused mainly by the choice of $g f$ values, and secondly by the model atmosphere adopted here. Nowadays MARCS models are usually emploied.

Synthetic spectra presented in Figs. 2-5 are in good agreement with observed ones. Differences between them can be caused by the following effects: (1) abundances of each element may differ from $[A / H]$, but large abundance optimization of selected elements (e.g. C, N, O, Mg,
$\mathrm{Fe}$ ), together with computation of appropriate model atmosphere, still overloads modern computers. However, for Arcturus we tried to compute abundances of individual elements with fixed main parameters (i.e. $T_{\text {eff }}, \log g,[A / H]$, $\left.v_{\text {turb }}\right)$, but the results are inconsistent, probably because we used limited spectral regions. (2) Line profiles may be affected by NLTE effects, although Drake \& Smith (1991) showed for $\beta \mathrm{Gem}$, that metallicities are not significantly affected by departures from NLTE. (3) Vertical stratification of some parameters, such us elements or microturbulent velocity, may affect line profiles (see for example Zboril 1996). (4) Finally, there may be errors in atomic constants, mainly oscillator strengths.

The present work mainly intended to show how spectrum fitting can be applied for the derivation of the atmosphere parameters of $\mathrm{K}$ giant stars. Another interesting comparison could be made while processing lines of each element separately, i.e. varying abundance of selected elements with constant atmosphere parameters (specially $[A / H])$, or, possibly, fitting lines of selected elements to obtain all atmosphere parameters. Moreover, discrepancies can be better discussed by the sorting of the lines by excitation potential, by ionization stages and by equivalent width. However, numerical tests showed that for such an analysis, spectra covering large wavelength intervals are required, but such study is beyond the scope of this paper.

Future research will be concentrated on the determination of atmosphere parameters of stars with lower metallicity (e.g. Pop II giants). This can help for a better understanding of the increasing discrepancy between the metallicities obtained for Arcturus. The results of these calculations will be part of a forthcoming paper (Krtička \& Štefl 1999, in preparation).

Acknowledgements. The authors are thankful to J. Kubát for kind help and to B. Plez for kindly providing model atmospheres. This research disposed of the SIMBAD database operated at CDS, Strasbourg, France. Tables determining of $T_{\text {eff }}$ $\log g$ and $[\mathrm{Fe} / \mathrm{H}]$ are taken from the catalogue of $[\mathrm{Fe} / \mathrm{H}]$ determinations (Cayrel de Strobel et al. 1997). We wish to thank our referee, F. Spite, for valuable remarks on this manuscript.

\section{References}

Abbett W.P., Beaver M., Davids B., et al., 1997, ApJ 480, 395 Barbuy B., Perrin M.N., Cayrel R., 1990, Rev. Mex. Astron. Astrofis. 21, 406

Bessell M.S., Castelli F., Plez B., 1998, A\&A 333, 231

Brown J.A., Wallerstein G., 1992, AJ 104, 1818

Cayrel de Strobel G., Soubiran C., Friel E.D., Ralite N., Francois P., 1997, A\&AS 124, 299

Cayrel R., Perrin M.N., Buser R., Barbuy B., Coupry M.F., 1991, A\&A 247, 122

Chavez M., Malagnini M.L., Morossi C., 1997, A\&AS 126, 267

Cowley C.R., 1996, in: Model Atmospheres and Spectrum Synthesis, 5th Viena - Workshop, Adelman S.J., Kupka 
F., Weiss W.W. (eds.). San Francisco, Astron. Soc. Pacific, San Francisco, ASP Conf. Ser. 108, p. 170

Cuisinier F., Buser R., Acker A., et al., 1994, A\&A 285, 943

Drake J.J., Smith G., 1991, MNRAS 250, 89

Fernandez-Villacanas J.L., Rego M., Cornide M., 1990, AJ 99, 1961

Gray D.F., The Observation and Analysis of Stellar Photospheres. Cambridge University Press, Cambridge, 1992

Gratton L., Gaudenzi S., Rossi C., Gratton R.G., 1982, MNRAS 201, 807

Gratton R.G., Ortolani S., 1986, A\&A 169, 201

Horn J., Kubát J., Harmanec P., et al., 1996, A\&A 309, 521

Hubeny I., Lanz T., Jeffery C.S., 1994, TLUSTY \& SYNSPEC - A User's Guide

Huber K.P., Herzberg G., 1979, Molecular spectra and Molecular Structure, IV. Constants of diatomic molecules. Van Nostrand Reinhold Company, New-York

Irwin A.W., ApJS 45, 621

Kurucz R.L., 1993, CD-ROM No. 18: ATLAS9 Stellar Atmosphere Programs and $2 \mathrm{~km} \mathrm{~s}^{-1}$ Grid

Kurucz R.L., 1993, CD-ROM No. 23: SYNTHE Spectrum Synthesis Programs and Line Data

Lambert D.L., Ries L.M., 1981, ApJ 248, 228

Leep M., Oke J.B., Wallerstein G., 1987, AJ 93, 338

Luck R.E., Challener S.L., 1995, AJ 110, 2968
McWilliam A., 1990, ApJS 74, 1075

McWilliam A., Rich R.M., 1994, ApJS 91, 749

Montes D., Martin E.L., Fernandez-Figueora M.J., Cornide M., De Castro E., 1997, A\&AS 123, 473

Piskunov N.E., Kupka F., Ryabchikova T.A., Weiss W.W., Jeffery C.S., 1995, A\&AS 112, 525

Plez B., 1997 (private communication)

Plez B., Brett J.M., Nordlund A., 1992, A\&A 256, 551

Škoda P., 1996, in: Astronomical Data Analysis Software and Systems V, Jacoby G.H., Barnes J. (eds.). San Francisco, Astron. Soc. Pacific, San Francisco, ASP Conf. Ser. 101, p. 187

Sneden C., Kraft R.P., Langer G.E., Prosser C.F., Shetrone M.D., 1994, AJ 107, 1773

Štefl V., 1985, Folia Fac. Sci. Nat. Univ. Purkyně Brunensis XXVI, 43

Štefl V., 1994, Scripta Fac. Sci. Nat. Univ. Masarykiana Brunensis 23, 67

Tomkin J., Lambert D.L., 1983, ApJ 273, 722

Valenti J.A., Piskunov N., 1996, A\&AS 118, 595

Wallace L., Hinkle K., 1996, ApJS 103, 235

Zboril M., 1996, in: Model Atmospheres and Spectrum Synthesis, 5th Vienna - Workshop, Adelman S.J., Kupka F., Weiss W.W. (eds.). San Francisco, Astron. Soc. Pacific, San Francisco, ASP Conf. Ser. 108, p. 170 